\title{
The Etiology, Pathogenesis, and Treatment of Vesical Calculus in Women.
}

By Chardes Greene Comston, M.D., Ex-Vice-President of the American Association of Obstetricians and Gynecologists; Member of the American Urological Association; Corresponding Member of the Urological Association of France, etc., Boston, Mass.

Pesical calculus is infinitely less frequent in the female than in the male, and in the former it might be considered quite a rare affection if one etiological factor was not prevalent, namely, the introduction of a foreign body into the bladder. The list of objects which have entered the bladder by way of the urethra and have given rise to infection, or have served as a nucleus for the formation of stones by precipitation of the salts normally contained in solution in the urine, is long. The calculus is in this case always secondary, that is to say, formed by phosphatic salts, and consequently is soft and friable, so that crushing is extremely easy.

I will first rapidly study the etiologic conditions and the pathogenic mechanism of resical calculi in women which develop primarily in an aseptic urinary apparatus, and secondarily in an infected bladder around a foreign body. After this I will rapidly consider the various operations for the removal of calculi, and will endeavour to form some conclusions relative to their indications and results.

Vesical calculi may be divided into two groups, according to whether they represent a primary formation from precipitation of the normal elements in solution in an acid or neutral urine, or secondarily in an infected, alkaline urine. Primary calculi are generally formed in the kidney, and upon reaching the bladder, if they become lodged there, increase in size by the deposit of strata of salts upon them. The primary form is composed of uric acid, sodium urate, calcium oxalate, or the bicalcic carbonate and phosphate, cystin or zanthin in varying proportions. Excepting cystin calculi, which are exceedingly rare, only about fifty cases being known, all others are very hard, and often render lithotrity quite difficult.

Secondary calculi, which are still called symptomatic, never form in an aseptic bladder. One will always find that at some time there has been an infection of the organ; the urine becomes alkaline and the tricalcic phosphates, amorphous phosphate of lime, ammoniaco- 
magnesia phosphates or urate of ammonium become precipitated and form a calculus around the foreign body which is responsible for the cystitis; the stone rapidly increases in size, and may even break up spontaneously. This variety of calculus is quite different from the primary form, being soft and friable, and is very oasily crushed and evacuated. Unfortunately the patients are exposed to a recurrence as long as the productive cause of the calculus remains, and from this fact there are special therapeutic indications, to which I will refer later on. For the moment it is sufficient to say that the foreign bodies should be removed, and the cystitis should be treated by aseptic instillations. In obstinate cases it may be necessary to resort to suprapubic cystotomy or vesico-vaginal incision, followed by a more or less prolonged drainage, but before doing this one should try the permanent catheter, which in producing a methodical drainage of the bladder also allows one to make topical applications, instillations and irrigations. From this it will be seen that there are two distinct types of vesical lithiasis, namely, one forming in an aseptic urinary apparatus, the other being simply a complication of vesical infection.

Primary calculi are very rare in women, for the same reason that gout, gravel and diabetes are exceptional in their sex. Some authorities have considered that the shortness and dilatability of the urethra, by allowing quite large calculi to pass through, are also reasons for their infrequency, but statistics are not in accord, and although Durand-Fardel found only 63 women out of a total of 326 subjects with gravel, Tuffer found 109 women and 94 men out of a total of 203 operations for stone of the bladder.

Many theories have been emitted in order to explain the formation of primary sand, gravel and calculi of the bladder. Generally speaking, acid renal or vesical lithiasis is a manifestation of the uric acid diathesis which, as is known since the writings of Bouchard, is one of the forms of tardy nutrition; the nitrogenous substances are incompletely burned, and are eliminated in the form of uric acid or the urates. Now, whenever the urine increases in acidity, the uric acid becomes precipitated, even when it is contained in normal amount, and likewise the urates become precipitated when the quantity of water is not sufficient to dissolve them. The abuse, or even the ordinary use, of certain vegetable foods, rich in oxalic acid, like tomatoes, asparagus, etc., produces an oxaluria, which is physiological in many subjects, but in those who are hereditarily predisposed may produce a lithiasis.

Why these salts dissolved in an acid and aseptic urine are not eliminated and why they become deposited and form calculi is 
perhaps to be explained by Ord's theory of the colloids, Ebstein and Nicolaier's histonecrotic theory, or the microbic theory. According to Ord the colloid substances which are found in normal urine (mucus) or when pathologic (albumen, sugar or blood) play, in relation to the solid matters in solution, the same part that gum plays with salts in solution in vitro. Thus the presence of these agglutinating substances is necessary for the formation of calculi, but this theory, I believe, is not generally admitted. Nevertheless, organic matter is necessary for the formation of vesical calculi, but the histonecrotic theory of Ebstein and Nicolaier gives a perfect explanation of how it may intervene in certain cases. These authorities having caused dogs to consume a certain amount of oxamide, emitted the hypothesis that this substance produces a necrosis of the epithelial cells of the kidney, which become the nucleus around which the salts are precipitated and form calculous concretions. Penzoltd also insists upon those foods which are commonly called "aggressive" for the kidney, which, by producing the detachment of the epithelium, favour the production of lithogenesis. This is at least the way that the formation of primary calculi is explained when they develop in a non-infected urinary apparatus and contain a nucleus composed of mucus and epithelial cells. It is probable, although quite impossible to prove in all cases, that this theory is the true one, and that a lesion of the epithelium often precedes a primary vesical calculus.

We should also refer briefly to the microbic theory which, at the present time, has been given up. Galippe, Kuhne and Waldeyer, having found agents of infection in primary calculi, thought that they were responsible for the formation of the latter, but the negative results of Ebstein, Albarran, and especially those of Chantemesse and Widal, have ruined this theory, from the fact that many women presenting primary calculi have never had an infection of the blood. To sum up, it will be seen that the question of lithogenesis is still most obscure, and many elements must be taken into consideration in order to explain the formation of primary calculi.

Secondary calculi represent the great majority of cases in women. Nine times out of ten one will be dealing with a phosphatic stone secondary to an old inflammation of the urinary tract. Under the influence of infection of the bladder the physical and chemical characters of the urine become changed and the salts are precipitated. Vesical infection is consequently the condition sine qua non. There has been a more or less intense cystitis which passes to the chronic state with permanent changes in the mucosa of the urinary reservoir. 
The necessary conditions for the formation of calculi, which are found with such difficulty in acid lithiasis, are here united; the nucleus around which the urinary salts become precipitated may be represented by pus globules, degenerated epithelial cells, false membrane, etc. Then, again, bacteria grow in the urine as in a tube of bouillon, decomposing the urea and rendering it alkaline, while the phosphatic salts and urate of ammonia become deposited and form a calculus which increases in size from the formation of parallel strata.

The question arises as to what may be the most frequent causes of an infection of the bladder. It is evident that a dirty catheter used for a traumatic or operative retention of urine, for example, may be the starting point of a very severe cystitis, which later on will become complicated by a secondary lithiasis. But it is well known that in order to become infected the bladder must be in a condition of morbid receptivity, and it is proved that the passage of a catheter is not always sufficient to contaminate a healthy bladder or one which is normally contractile. Other causes are necessary, such as traumatism, retention or congestion. For example, a catheter is passed on a woman without sufficient aseptic precaution, but for all that no aymptoms of cystitis will develop, because the bladder will easily expel all the bacteria introduced on the instrument. It would not, however, be the same if the mucosa is injured, even slightly, or if the bacteria are introduced in large numbers into the bladder, and this brings us to the important part played by foreign bodies.

Foreign bodies of the bladder in women are very frequently encountered, usually being introduced during the mancourres of onanism; they then give rise to an infection of the organ, which prepares or at once sets up a severe form of cystitis. Quite frequently their presence in the urinary reservoir is easily tolerated for a number of years, but some day, under the influence of congestion or retention, a cystitis becomes declared, and the foreign body forms a nucleus around which calcium salts become precipitated, and a secondary calculus is rapidly formed. Some authorities have upheld that vesical calculi in women are always due to a foreign body in the bladder, but without going so far, it may be said that this is frequently the case, and this origin should always be suspected when a calculus of the bladder is met with in a young woman.

The foreign bodies that may be encountered in the bladder vary considerably, but for convenience sake they may be classed into four groups-(1) foreign bodies intraduced for therapeutic purposes, such as sounds, pieces of rubber catheter, nitrate of silver, bits of various 
instruments which become broken off in the bladder; (2) foreign bodies introduced during the act of masturbation, such as pins, hairpins, boot buttoners, knitting needles, pencils, bits of wood, injection syringes, pipe stems, cigarette holders, fruit stones, metallic balls, sealing wax, pocket knives, keys, fish bones, etc.; (3) foreign bodies coming from without by traumatism, such as various forms of projectiles, pieces of clothing and splinters of bone; (4) foreign bodies coming from neighbouring organs by the intermediary of a perforation or fistula, such as pessaries, which have perforated the vesico-vaginal septum and penetrated into the bladder, alimentary debris, intestinal worms coming from the rectum, and, lastly, debris coming from dermoid cysts, such as hair, teeth and bits of bone.

In the majority of cases the hairpin is more usually introduced during manœuvres of masturbation; consequently it is in the adult age, between twenty and forty years, that these are met with. When once they reach the bladder foreign bodies become accommodated according to certain well-determined positions. If they are small they fall into the fundus; if they vary in length from 6 to 9 centimetres they become placed transversely following the greatest diameter of the bladder; if they measure more than 9 centimetres they are placed either vertically or obliquely. When they remain in the bladder they usually set up a simple cystitis, but in other cases when one of their ends press upon the bladder wall an ulcerative process is set up which extends deeper and deeper until perforation of the vesico-vaginal septum takes place. Under these circumstances the foreign body may be eliminated.

When the foreign body remains in the bladder it soon becomes incrusted with salts, and it is well known with what rapidity a permanent catheter in cases of infected prostatic bladders becomes covered with phosphatic concretions. These incrustations generally begin on the broadest part of the foreign body, and extend to the ends. A calculus thus formed, over a hairpin for example, has an oval shape with more or less rounded poles. However, when the nucleus is a hairpin, a pencil or a pointed body the extremities frequently remain uncovered and offer great danger to the wall of the bladder in which they become buried, sometimes causing suppurating ulcerations which give rise to extremely severe pain. Secondary calculi developing on foreign bodies are susceptible of attaining very large dimensions, and may become complicated with a fatal uretero-pyelonephritis, as in all instances where the bladder is seriously infected.

Large calculi are often eliminated by way of the vagina after 
having acquired very large dimensions, and they have been known to weigh as much as $12 \mathrm{oz}$. Urinary incontinence by a fistula is then quite frequent, but not always fatal; thus, for example, Agnew has related a case of a calculus the size of a goose's egg, which was eliminated, although no incontinence was present, and Keyes met with a case in which the circumference of the calculus measured five inches.

Such, briefly, is our knowledge at the present time of the etiology and pathogenesis of vesical calculi in the female, and we will now consider their treatment, but before doing this we will rapidly sum up what has already been said. Vesical calculi in women are usually secondary manifestations of an infected bladder, while the introduction of foreign bodies is often the origin of the infection, as well as the nucleus of the calculus. Primary calculi are extremely rare, and are manifestations of the uric acid diathesis and of oxaluria. They are formed by precipitation of the acid salts in excess in the urine, around necrosed epithelial cells, clumps of white or red blood cells, or mucus, which form nuclei, around which the mineral matter becomes deposited.

The logical treatment to apply to calculi of the bladder is naturally surgical, and by this means the patient is placed out of danger from the numerous complications which may arise, although, however, a few words on the medical treatment may not be out of place.

Medical treatment can only be applied in cases of acid lithiasis, that is to say, to uric acid or oxalic acid calculi. In consists in diet and the administration of certain medicines.

The use of dark meat, venison, spiced and salted meats and sea fish should be particularly avoided, and acid regetables, such as tomatoes, asparagus, etc., which contain oxalic acid should be proscribed. On the contrary, green vegetables may be allowed. Light white wine, beer and cider may be permitted because by their diuretic action they eliminate the organic residue and irrigate the urinary tract. Secondly, the nutrition should be made as accurate as possible, so that the nutritive exchanges may result in the total elimination of the uric acid. And for this purpose properly directed bodily exercise, hydrotherapy, dry friction and massage will contribute towards this end very greatly. But all this should be aided by alkaline medication, because it exercises a very efficacious action, although as yet badly understood as far as its intimate mechanism is concerned, but the effects of which are easily rerified even several months after stopping the administration of the alkalines. It has 
only been recently known that the latter increase the production of urea, which is the most advanced form of oxidation.

Bicarbonate of soda and the salts of lithia are the alkalines the most employed, but of late years new drugs have come in vogue, such as benzoic acid and the benzoates, urotropine, piperaztne, lycetol, etc.; and, lastly, I would mention a few of the innumerable mineral waters which act by throwing out the urates from the tissues, such as Vichy, Royat, Pougues, Ems, or as diuretics we have Evian, Vittel, Contrexeville, etc.; others stimulate the hepatic functions by the intermediary of the digestive tract, namely, Carlsbad, Brides, Marienbad, Kissingen, Plombières, Bourbonne-les-Bains, etc. As to curative treatment by so-called dissolvents, it has remained as a faint hope. Although the various lithontriptics have certainly an active influence on nutritive exchanges they nevertheless are powerless to dissolve a vesical calculus, and experiments which have been realised in vitro cannot be applied to the living, because the substances employed cannot be tolerated by the bladder.

Two methods dispute surgical favour in the treatment of vesical calculi, namely, cystotomy by which the stone is at once removed, and lithotrity. In the female, whose urethra is short and easily dilated, a third method may also be discussed, namely, dilatation. It is well known that quite large calculi are frequently expelled by the female urethra, and for many years surgeons have endeavoured to imitate nature. In $1817 \mathrm{Sir}$ Astley Cooper vigorously defended this mothod, which, for the first time had been advised, but without result, by Tollet in 1708 in his "Traité de la Lithotomie." According to the experiments of Hylord, the neck of the bladder can easily reach the diameter of 10 millimetres without becoming injured, but it is well known that it can be distended far beyond these limits, and that under complete narcosis, by introducing one finger after another the diameter may be made to reach as much as 3 centimetres. It can be readily understood that, under these circumstances, small calculi may quite possibly be removed in this manner, and spontaneous expulsion shows that, under the mere contraction of the bladder, this dilatation is quite possible without any ill result. Thus, for example, Sir Astley Cooper records the case of an 18 year old girl, who in 10 minutes expelled a calculus $2 \frac{1}{2}$ inches wide and $1 \frac{1}{2}$ inches long, while Cabot has related the case of two women, who passed respectively a calculus the size of a pigeon's egg, and the other one the size of a hen's egg. These cases are instances taken haphazard among a very large number.

The technique of dilatation is simple. Anæsthesia is necessary 
because the muscular fibres can be stretched without reacting, and it is an easy matter to remove the calculus from a paralysed bladder. The patient is placed in the lithotomy position, and the urethra dilated either with or without incising the meatus. All dilators should be discarded and the fingers alone should be used, because one can more easily judge of the tension produced and avoid more or less the rupture of the sphincters. The use of Hegar's sounds may also be resorted to. Some operators have stated that the urethra could be dilated without permanent incontinence following, up to a diameter of 3 inch, but if this is done there is great fear of lacerating the mucosa and spongy tissue, which, even when superficial, opens wide the door to infection.

The anatomical position of the bladder makes it easy to reach by the vagina, vulva or abdomen, from which is derived four procedures of cystotomy, two of which only have resisted the test of time, and these alone will be considered, namely, colpocystotomy and suprapubic cystotomy. Colpocystotomy, which has in turn been advised and abandoned since Fabrivius de Hilden, who only employed it in women having a cystocele, is to-day considered an excellent method, which will not be complicated by incontinence if the neck of the bladder is not involved, and the patient will recover without a fistula if the incision is properly sutured. There is no very great difficulty in the technique. All that is necessary is to introduce a male sound through the urethra, preferably a grooved one, with which the lower wall of the urinary reservoir is pressed down, holding the instrument directly in the median line. Beginning back of the urethra, the vesico-vaginal septum is incised to the extent of 3 or 4 centimetres, just giving space enough to admit two fingers or an instrument for the removal of the stone. Should this opening not be large enough for the easy passage of the calculus through it, it can be gently dilated until sufficient space is obtained. If the bladder is infected, as is usually the case, it can be efficiently drained by this opening, and if there is a tendency to too rapid closure it may be kept open by occasionally dilating it with the finger or an instrument. Drainage is particularly indicated in serious inflammatory lesions of the bladder, when the interior of the organ will be frequently found lined with a layer of phosphatic deposit, which should be removed by a methodical and prudent scraping. If necessary drainage tubes may be employed, because it is well known that these incisions have a remarkable tendency to spontaneously close very quickly. Irrigations may be done through the drainage tubes and by the urethra, and as soon as possible, that is to say, when the condition of the bladder 
will allow it, the opening may be closed by the ordinary technique employed for vesica-vaginal fistula.

No matter what changes in detail each surgeon has made in this operation, it generally succeeds without causing incontinence or a vesico-vaginal fistula. If a suture should cut through, resulting in a small orifice communicating with the interior of the bladder, it will usually close up quite quickly by the use of antiseptic vaginal irrigations, but should the small fistula persist its borders are to be freshened and sutured according to the usual methods.

Suprapubic cystotomy consists in opening the bladder by way of the abdomen. The operation is extremely easy in the male, but I have found it more difficult in women. In the first place, it is a difficult matter to distend the female bladder with either liquid or air, and one is obliged to have the urethra compressed by an assistant after the bladder is filled until it is opened above. As I never use Petersen's bag in the rectum the question will not be brought up here. It should be pointed out that the suprapubic incision will allow one to make a very much larger opening into the bladder than can be done in any other operation; and it is possible to extract with the fingers foreign bodies or large calculi which have become wedged into a diverticulum without tearing or hardly scratching the mucosa. Then again, this operation gives a perfect view of the interior of the bladder, and if there is any indication, any lesions found therein may be directly treated under the control of the sight.

If the bladder is aseptic, a condition which is very rare and only met with in cases of uric acid calculus, the bladder may be closed at once. But if, on the contrary, it is infected, it should be drained.

Lithotrity also presents more difficulty in the female than in the male, and this argument has been brought up in order to reduce the indications of this operation in favour of cystotomy. It is quite true that the impossibility of filling the female bladder under the influence of narcosis is a great hindrance to crushing, but for a surgeon who is used to the operation it may be performed with safety in an empty bladder. It is also true, as has often been repeated, that the female bladder is large and lax, deprived of any fundus in which the fragments tend to drop after crushing, as takes place in the rectro-prostatic fundus in the male. For this reason the bits of calculus slip away from the jaws of the lithotrite, but this is in reality a difficulty more theoretical than real. In point of fact, in the female it is not necessary to reduce the calculus into such small fragments as in the male on account of the shortness of the urethra, as well as its easy dilatation, which renders the extraction an easy 
matter. Aspiration of the crushed calculus is not necessary in the female, and does not need to be preceded by so careful a crushing as in the male; large pieces are spontaneously evacuated, or may even be removed by long slender dressing forceps.

It may consequently be seen that these objections which are reproduced everywhere are not of sufficient value to incriminate lithotrity in the female, and we would repeat that the difficulty of filling the bladder with liquid and the absence of a fundus in which the pieces may collect are of little importance in practice, because the crushing need not be pushed very far on account of the easy extraction of the fragments by way of the urethra. If, beside these considerations, it is remembered that lithotrity is a very simple and harmless operation, that patients readily accept it when they will frequently refuse a cutting operation, and that the post-operative convalescence is reduced to two or three days in bed, while the vesico-vaginal or suprapubic cystotomy requires at least a fortnight and exposes the patient to some little risk, it may certainly be said that lithotrity merits to be considered as the best mode of treatment of vesical calculi which are too large to be removed through the dilated urethra.

The patient is placed in the lithotomy position with the pelvis raised, or a slight Trendelenburg position may be used. The lithotrite should be held almost vertically and made to depress the vaginal wall just behind the neck of the bladder, so that a declivous portion will be made in which the calculus may be seized. It is well to inject 100 or 150 grams of liquid into the bladder. The crushing should be done in this declivous portion, the female branch of the instrument being fixed and depressing the vesical vaginal wall, the male branch alone being made movable. In those cases where the cervix uteri produces a lateral pocket one should endeavour to propel the calculus into it. Aspiration should always be preceded by irrigation with one of Bigelow's large straight catheters, which is easy to direct to the point where the débris are to be found. The aspiration itself is proceeded with as in the male, and if some fine pieces remain in the bladder they will usually be easily expelled. Vesical irrigations should be continued for several days after the operation.

The indications and the comparative value of dilatation, cystotomy and lithotrity cannot be based upon any statistics which we now possess, because it would be unjust towards the latter operation to consider death from pyelo-nephritis which had existed before the operation as due to this interference. We will simply limit our 
remarks to the advantages and inconveniences belonging to each of these operations, and endeavour to draw some conclusions as to the respective indications for each. Urethral dilatation, vesico-vaginal or suprapubic cystotomy and lithotrity are the four operations which should be considered. They all require general narcosis, which is a common point and exposes them all to the accidents of anæsthesia, but this is the only respect in which they are alike, and in all others they are entirely different.

Rapid dilatation is followed for a certain time by incontinence of urine, and unless one has lacerated the sphincter this accident will be recovered from, and is perhaps the only black point in this operation; but if the calculus be a large one or even of medium size it cannot be extracter by this route, and as a rule it may be laid down that dilatation may be resorted to in cases where the stone has not more than $2 \frac{1}{2}$ centimetres in diameter. It is formally contraindicated in girls under 15 years of age on account of the small calibre of the urethra, as well as the close approximation of the pubic bones which hinder the manouvres of extraction. It cannot be employed in very elderly women, because in them the urethra has lost its suppleness and has become rigid, so that it cannot be dilated sufficiently without rupturing it. In adults afflicted with incontinence of urine due to a weak sphincter, dilatation cannot be resorted to because it would only aggravate the pre-existing infirmity. With these exceptions, when the calculus is not large, dilatation is an excellent operation, and if carried out with care may be resorted to with a good chance of a perfect result. If, after dilatation, the stone is found too large to be removed intact, a few strokes of the lithotrite will be sufficient to break it up into fragments, which then can be removed by the urethra with forceps or the fingers.

Colpocystotomy will give a working space of about 3 centimetres, and by spreading the borders of the wound apart the dimensions of the opening will be made still greater. The production of a persistent fistula is not to be feared with the present methods of operating. This operation has the great advantage of never giving rise to incontinence of urine from a lesion of the sphincter, and is very rarely followed by a fistula when the technique has been properly carried out. This method, which is considered by Emmet and many other American surgeons as the best means of treating severe forms of cystitis on account of the easy drainage it gives, and which is at the same time permanent, may be applied to large calculi complicated with sorious and long-standing inflammatory lesions of the bladder, becausu, after removal of the stone, it allows one to continue the 
drainage and at the same time apply topical treatment to the vesical mucosa.

There exist, however, certain contra-indications, the first of which is a narrow vagina which will prevent the requisite manœuvres, and under the circumstances one should not hesitate to resort to the suprapubic route. The American Text-Book of Surgery makes the statement that lithotrity is considered the best treatment for vesical calculus in the fomale, and is only contra-indicated by the large size and density of the stone as well as by the intensity of the vesical inflammation, under which circumstances one should resort to colpocystotomy. It also advises an immediate suture of the incision if no cystitis is present, but the fistula will be allowed to remain if the bladder is infected. It also makes the statement that suprapubic cystotomy has very limited indications in the female. Personally, I believe that the suprapubic incision in women should in principle be rejected or at least reserved for extremely infrequent cases.

Many operators, basing their remarks especially on their own practice, have discussed the advantages and the inconveniences of colpocystotomy and litholapaxy, and have arrived at very varied conclusions; but it would seem to me that common sense would allow one to admit in principle that in the female, as in the male, lithotrity is an operation practically devoid of any danger when properly carried out, and in the female will be followed by a more rapid recovery than can be obtained by either the vaginal or suprapubic incision. I have already pointed out the difficulties of the latter operation in the female, but further than this it is no more to be feared than in the male, and I am a warm partisan of this operation in the male in suitable cases, believing that in simplicity and results it is far superior to any other modern technique, but in the female I am not prepared to make this statement. I have already pointed out the indications for colpocystotomy, and it is well known that by a well-directed technique the operative difficulties of lithotrity have been remedied.

If we now consider the operation in itself, it at once becomes evident that lithotrity presents very great advantages over cystotomy. It is, in the first place, more readily accepted by patients, and this is not a small point to be taken into consideration. I do not believe that there is any difference in the prognosis of the two operations as far as mortality is concerned, but as to the time of convalescence it is reduced to two or three days in bed without pain, incontinence or dressings, and the patients can return to their ordinary mode of 
living within five or six days after the operation. Now, in the case of cystotomy, either by the vagina or the abdomen, there is the great nuisance of drainage, and it is hardly possible to promise these patients less than three weeks in bed.

Now, as we have pointed out, vesical calculi in the female are usually secondary, and for this reason they are soft, and I have also insisted on the facility with which even very large fragments of stone can be spontaneously passed; consequently the best conditions are found united in lithotrity to give the result expected from it. Even when one is dealing with a foreign body incrusted with phosphates this operation is sufficient, because by a few turns of the screw of the instrument the stone is broken up and the foreign body, if not too large, may be removed by the urethra. In the second volume of the System of Surgery, Morris states that, in his opinion, lithotrity is rarely necessary in women on account of the physical conditions presented by the urethra, and that when the stone is too large to be removed in this way crushing is the best operation. He goes on to say that in females having large calculi they may be removed by colpocystotomy followed by immediate suture if the condition of the bladder allows it. But in young women litholapaxy should be systematically resorted to. In Dic chirurgischen Krankheiten der Harn und mannlichen Geschlechtsorgane Guterbock takes the same view as far as lithotrity is concerned in the large majority of cases, and he believes that suprapubic cystotomy should be applied only in cases of young girls having very large and hard calculi.

To sum up the treatment, it may be said that as far as the medical part is concerned, it consiste in careful hygiene and alkaline medication, having for end to stimulate the nutritive exchanges and favour the elimination of waste products. The surgical treatment consists in urethral dilatation, vesico-vaginal or suprapubic cystotomy and lithotrity. Each of these operations has its advantages and its indications. Dilatation is not possible in children, and will not allow of the extraction of calculi exceeding 3 centimetres in diameter. Suprapubic cystotomy should be reserved for cases of large calculi in young girls. Colpocystotomy is to be resorted to in cases where the stone is large or extromely hard or is developed around foreign bodies and becomes lodged in the bladder, also in cases of vesical infection. Lithotrity would seem to be the method of choice when the stone is friable and of medium sie. It is followed by the spontaneous expulsion of fragments should any be left behind after the operation is completed and allows the patient to resume her ordinary life within a few days if preceding inflammatory complications of the bladder do not require drainage of the organ. 